\title{
Tele-operated robotic arm and hand with intuitive control and haptic feedback
}

\section{Monica Dascalu ${ }^{1,2}$, Mihail Stefan Teodorescu ${ }^{1}$, Anca Plavitu ${ }^{2,3}$, Lucian Milea ${ }^{1}$, Eduard Franti ${ }^{2,4}$, Dan Coroama ${ }^{1}$, Doina Moraru ${ }^{1}$}

${ }^{1}$ Faculty of Electronics and Telecommunications, Politehnica University of Bucharest, Bucharest, Romania

${ }^{2}$ Center for New Electronic Architecture, Research Institute for Artificial Intelligence, Bucharest, Romania

${ }^{3}$ Faculty - Exact Sciences and Engineering, Hyperion University, Bucharest, Romania

${ }^{4}$ National Institute for Research and Development in Microtechnologies, Bucharest, Romania

\section{Email address:}

monicad@artsoc.ro (M. Dascalu), mihait@artsoc.ro (M. Teodorescu), ancap@artsoc.ro (A. Plavitu), edif@artsoc.ro (E. Franti), lucian@artsoc.ro (L. Milea), dcoroama@artsoc.ro (D. Coroama), doinam@artsoc.ro (D. Moraru)

\section{To cite this article:}

Monica Dascalu, Mihail Stefan Teodorescu, Anca Plavitu, Lucian Milea, Eduard Franti, Dan Coroama, Doina Moraru. Tele-Operated Robotic Arm and Hand with Intuitive Control and Haptic Feedback. American Journal of Aerospace Engineering. Vol. 1, No. 4, 2014 , pp. $21-27$. doi: 10.11648/j.ajae.20140104.11

\begin{abstract}
The paper presents a robotic arm having as end effector an anthropomorphic hand and its control system. The robotic arm and hand are controlled using a Complex Interactive Control Glove (CICG) and operator joint sensors. The robotic hand imitates the finger and joint movements of the human operator. The anthropomorphic hand sends pressure feedback from a pressure sensor array mounted at the robotic hand's fingers and palm to the human operator wearing a Complex Interactive Control Glove that comprises haptic actuators. The pressure exerted by the robotic hand on various objects is perceived as vibrations on the corresponding hand area of the human operator. The robotic arm adjusts its position in correlation with the human operator's arm, placing the end effector at the right position, corresponding to the operator's hand. Data for the movement of the robotic arm are collected from the movements of the human operator by means of three joint sensors placed on the shoulder, elbow and hand wrist. Targeted applications of the tele-operated robotic arm and hand with intuitive control and haptic feedback include all situations where a human-like operation is needed in a hazardous or remote environment: space environment, operations executed in toxic atmosphere, working in high-radiation level environments, marine applications. In such cases, the robotic hand and arm that are executing the same movements as the human operator can replace the actual human operator. This will control the robotic arm form a safe, possibly remote, environment, and will be able to process the haptic feedback of the systems.
\end{abstract}

Keywords: Robotic Arm, Robotic Anthropomorphic Hand, Haptic Feedback, Complex Interactive Control Glove, Hazardous Environments

\section{Introduction}

In the past years, robotic arms and robotic hands have gained more and more attention due to their diversified applications in the industrial field. Significant advances were also made in medical domain, military applications, marine and space exploration, and even entertainment and home applications. Robotic arms usually use as end effector a task-specific designed gripper. Robotic arms are nowadays available in a variety of realizations, from industrial types, with ranges, degrees of freedom, speed and force by far exceeding the ones of a human arm, to anthropomorphic robotic arms that partially or nearly fully reproduce the capabilities of a human arm.

Some basic characteristics that define the performance of a robotic arm are: number of limbs and joints, degrees of freedom, force, speed, accuracy, repeatability, performances of the control system [1]. A robotic arm has attached an end effector that is specifically designed to interact with the environment and its exact nature depends on the application of the robot. Usually the end effector consists of a gripper or a tool and in some cases of an anthropomorphic hand. When the end effector is a tool, it serves various purposes, such as spot welding in an assembly, spray painting where uniformity of painting is necessary and for other purposes where the working conditions are dangerous for human beings. Surgical 
robots have end effectors that are specifically manufactured. In this case the robot arm can be used only for that particular purpose, any other operation requiring a tool change, if possible.

When referring to robotic prehension there are four general categories of robot grippers.

- Impactive - claw-like grippers

- Ingressive - needle type gripper

- Astrictive - suction gripper

- Contigutive - require glues, freezing or other types of adhesion.

The most known impactive grippers consist of two, three or even five fingers. They can have different degrees of freedom (DOF) and can be used in different environments exerting various movement types and forces. A particular case occurs when the gripper is an anthropomorphic hand, which is useful or even imperative when the robotic harm has to perform operations with human-like dexterity, replacing a human in hazardous environments, hard-to-reach places or wherever a life-threatening or risky situation appears. One of the key issues in the field is the control system, mainly the human-robot interaction [2] [3].

Difficulties an challenges encountered in designing robotic anthropomorphic hands comes first from the decomposition of continuous natural movement of the hand in distinct components that can be imitated by the artificial hand. Other typical limitations occurs in reproducing/implementing the feedback data from a human hand in an artificial one. Tactile, temperature, position and force feedback would be very useful in controlling the hand and taking the right decisions, but even very modern sensors cannot cover the whole range of natural sensory feedback.

Different methods are proposed in scientific literature in order to improve the feedback of a robotic hand [4]. A number of spots for tactile feedback can be applied to the robotic hand and the force feedback can be read from the power absorbed by the driving motors. Many other methods to gain sensory feedback are used, considering also accuracy and cost. The computing power needed to process all the incoming data and run the implemented software algorithms is also to be considered.

Another important challenge regards the control method of the anthropomorphic hand. The dynamics of the human hand can only be partially modeled and dexterity implies brain-like computing power, by far not available nowadays. Automatized procedures for limited fixed tasks or a human operator for unforeseen operations are the best choice for controlling the robot hand and arm. Preprogrammed sets of movements can be stored in a computer memory and put in use for the appropriate situations. If a human operator is implied, that remotely controls the robotic hand attached to a robotic arm, several control methods have been researched and applied [5] [6] [7] [8].

The traditional method is controlling the robotic limbs with a joystick-type controller and/or a keyboard. This is also the most difficult method requiring a specific training for the operator since the movements of the operator and the desired movements of the robot differ significantly. Consequently, no force or pressure feedback can be given directly to the operator and usually few or no warning signals are used.

Another much more intuitive method is to have a duplicate robotic arm that is physically moved by the operator. All the movements of the joints are sensed and reproduced in the operating remote arm, as accurate as possible and in real time. This method implies mostly the control of a robotic arm and a rough end effector, but it can also offer force feedback to the operator, if constructed accordingly.

The operator driven arm and hand can also take the shape of an exoskeleton covering the human operators shoulder, arm and even fingers, which could produce maximum accuracy in reproducing the operator's movements and also some force feedback, but it implies a mechanical and electrical complexity difficult to deal with.

Other recently reported control methods are position and motion detectors for the operators' arm or even mind controlled robots, but research is only at the beginning [9] [10]. The sensors used to track movement and position can be accelerometers, Hall Effect sensors, potentiometers, tension sensors or others.

For teleoperated robots, haptic feedback from the robotic hand implies using tactile sensors that reproduce the sense of touch, sending the corresponding signals to the human operator or control system and again translating the signals in visual, audible or preferable tactile stimuli sensed by the operator [4]. The haptic actuator provides tactile sensing by means of vibration to the human operators hand. The vibrations can be produced by different actuator types.

The simpler and cost effective option is the Eccentric Rotating Mass vibration motor, or ERM. Linear resonant actuators (LRA) are widely used in haptic feedback applications because of their low response times and feasibility.

The paper presents a robotic arm having as end effector an anthropomorphic hand controlled using a Complex Interactive Control Glove (CICG) and operator joint sensors. The robotic hand imitates the finger and joint movements of the human operator and sends feedback. The pressure exerted by the robotic hand on various objects is perceived as vibrations on the corresponding hand area of the human operator. The robotic arm adjusts its position in correlation with the human operator's arm, placing the end effector at the right position, corresponding to the operator's hand.

The architecture of the system, including control system and the robotic arm, put together for the first time different ideas from our own research and scientific literature. The novelty of the system consists mainly in control of the robotic arm through imitation of the operator's arm (see fig.1), based on flex sensors and sustained by complex processing algorithms. The structure is capable to execute fluently sequences of complex high precision movements that imply combinations of simultaneous movements of the shoulder, the elbow and the wrist. The computing algorithms that process the signals from the sensors and generate the commands for the robotic arm and hand are also new and original - they are 
only intuitively explained in this paper.

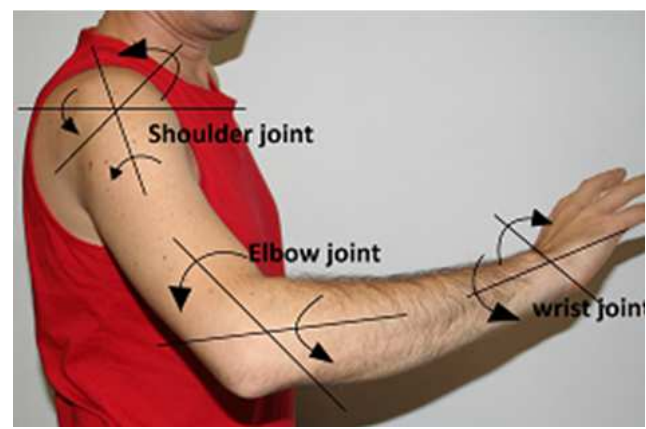

Fig. 1. Movement types of the human arm and hand

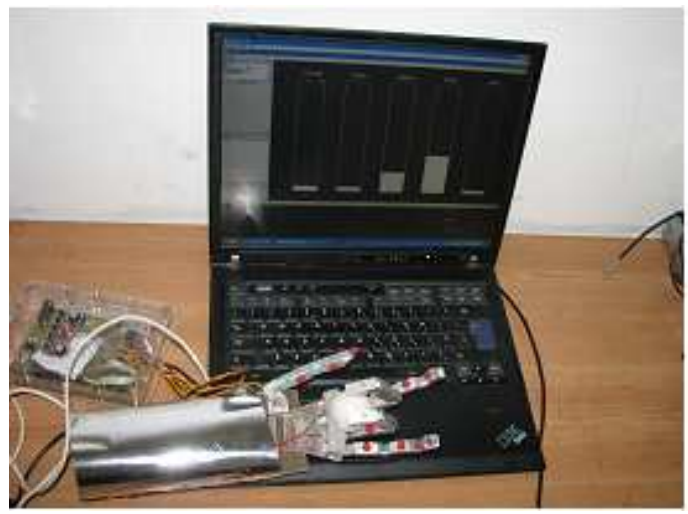

Fig. 2a. First anthropomorphic hand prototype - mechanical structure

\section{The Mechanical Structure that Replicate the Human Arm and Hand in the Robotic System}

The mechanical robot arm and end effector, as presented in this paper, resembles in principle with the anatomic structure of the complete human arm; it should be able to imitate the main movements of the human arm as shown in fig.1.

The robotic arm consists of a number of joints and links. The mechanical joints are usually restricted to one DOF, which results in simpler control, mechanics and kinematics. A robotic arm attempting to reproduce a human arm consists of 2 moving links connected to a fixed base and three joints, forming a simple kinematic chain. The joint at the base represents the human shoulder, the joint that unites the two links represents the human elbow and the joint situated at the end of this chain connects the end effector, eventually an anthropomorphic hand.

The human hand is considered to have 27 degrees of freedom (DOF): 3 for extension and flexion and one for abduction and adduction, 4 in each finger; the thumb has 5 DOF and there are 6 DOF for the wrist. For the construction of a robotic hand usually some simplifying assumptions are made from the start, like the thumb is considered independent from the other fingers or the adduction/abduction of the finger joints are independent, which correspond to individual control of the fingers.

The mechanical structure of the anthropomorphic hand is usually a simplification of the human hands structure, being constructed of the same number of joints and links, but having 7 - 20 DOF. Each joint has 1 or 2 DOF.

Upon the completion of the present work, three mechanical structures of the robotic hand were successively adopted and tested. The robotic hand structure resembles the human anatomic structure of the human hand using one DOF joints, which allow efficient grasping of objects maintaining a firm grip. The prototypes described in this section were essentially used in developing the processing algorithms and implementing the control unit able to generate the complex movements of the artificial arm and hand.

The first prototype is presented in fig. 2 a without sensors attached, as it first was put in use, and in fig. $2 \mathrm{~b}$ with pressure sensors attached to the phalanxes and control system.

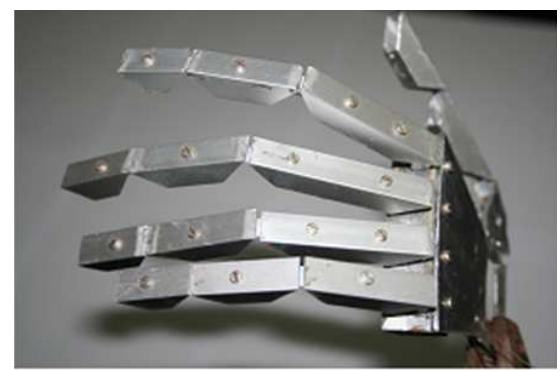

Fig. 2b. First anthropomorphic hand prototype with pressure sensors.

The second developed prototype started from a simple plastic replica of the human hand. Control strings and advanced pressure sensors were attached to the phalanxes, as shown in fig. $3 \mathrm{a}$, resulting in the robotic hand structure presented in fig. $3 \mathrm{~b}$.

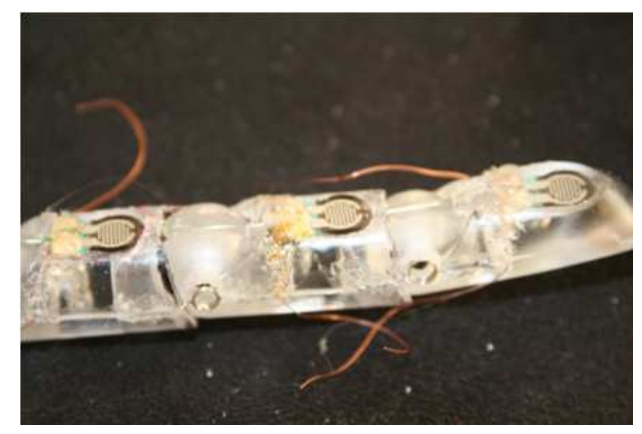

Fig. 3a. Attaching movement strings and pressure sensors to the mechanical structure

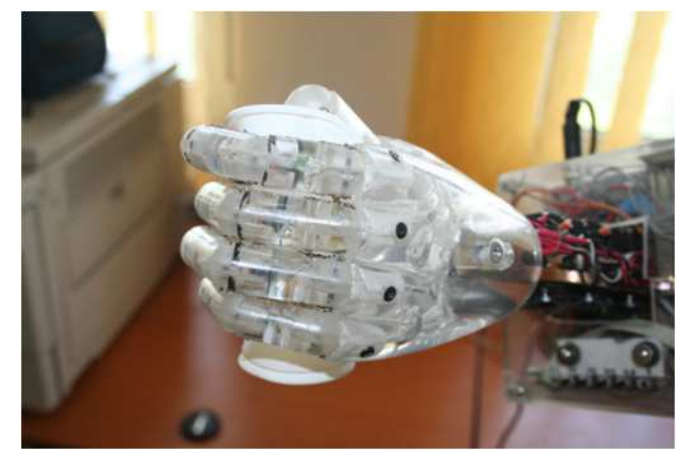

Fig. 3 b. Prototype 2 of the robotic hand with haptic feedback 


\section{Block Diagram of the Robotic System}

Our goal is to design a teleoperated robotic arm and anthropomorphic hand with haptic feedback, controlled intuitively by a human operator. The movements of the operator's arm and hand will be exactly reproduced by the robotic system, which provides tactile feedback to the human operator regarding the pressure confronted with. This will enable the possibility of complex and high precision manipulation in unfriendly and hazardous environments, without exposing the human operator to risks. The human operator is equipped with three joint sensors and a control-glove, that will assure performing high-precision tasks with almost no preliminary training required for the operator (the robotic hand having all five articulated and driven fingers and allowing human-like, complex maneuvers).

The block diagram of the implemented robotic system is presented in fig. 4. The robotic arm and hand are remotely controlled by the human operator and imitate his arm and hand movements. The anthropomorphic robotic hand is equipped with a pressure sensor array of 20 sensors distributed on the fingers and palm.

The sensors transmit the force encountered by the hand during manipulation by means of feedback signals to the control unit. The control unit manages to send in real time the sensor signals to a haptic actuator array that comprises also 20 actuators attached to the Complex Interactive Control Glove that is warn by the human operator. The operator perceives the pressure exerted on the robotic hand through vibrations executed by haptic actuators placed on the human hand in the same position as the pressure sensors on the robotic hand. The amplitude of the vibrations is proportional to the pressure level on the robotic hand.

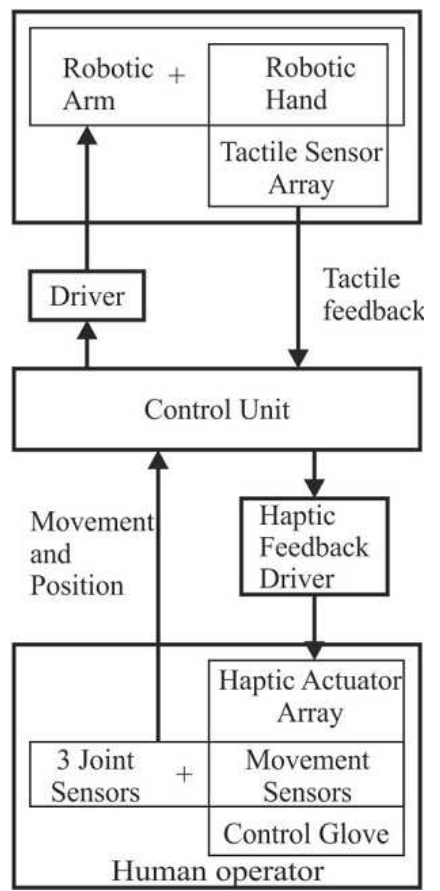

Fig. 4. Block diagram of the robotic system
The human operator uses a Complex Interactive Control Glove and three joint sensors to control the movements of the robotic arm and hand. The three joint sensors are situated on the shoulder, the elbow and the wrist and sense their movements. The Complex Interactive Control Glove comprises the tactile actuator array mentioned above and also movement sensors on every finger. The movement and position signals from the joint sensors and the glove are sampled by the control unit, processed and then control signals are sent to the drivers of the robotic arm and hand.

The control function is distributed into sub-systems of lower complexity for each component, simultaneously creating the possibility of implementing the self-management function for achieving the global control. Thus, a hierarchical and distributed architecture for the coordination and control system of the mobile elements in the robotic hand is devised. The main advantage this architecture is the very short reaction time to the control commands the device will receive from the operator, thus ensuring real-time functioning of the mobile elements in the robotic system.

The control unit analyses the movement and position signals from the operators hand and limits the movements of the robotic arm in one of the following situations:

- If the position of the robotic arm and hand that will result consequent to the next movement (performed during a sampling period) will exceed the preprogrammed boundaries. This function is meant to avoid collision between the robot hand and nearby obstacles.

- If the acceleration or speed of the movements executed by the operator exceeds certain values for the three joints and for the fingers. Even if mechanically the arm and hand can perform at the same acceleration or speed as the human operator, some speed levels are considered dangerous for the integrity of the robotic system or for nearby working humans or equipment. Also the possibility of losing grasp on the manipulated object has to be considered due to inertial forces.

The Control unit also analyses the signals from the tactile array and automatically stops the hand grasping movements in one of the following situations:

- If the pressure exerted on an object reaches a preprogrammed value, then this value is maintained to keep the grasping force and is not increased in order to protect the manipulated object from deterioration.

- If the pressure on a specific sensor or sensors exceeds a certain value and also is much higher relative to the pressure on nearby sensors, the grasping force is slightly reduced in order to protect the sensor or the robotic hand from deterioration.

Repetitive movements or operations can be programmed and triggered as desired. During their run, the robotic system functions without the intervention of the human operator performing certain tasks. Thus, the robotic system can function in one of the following three control modes:

- complete human control,

- computer control, 
- combined human and computer control.

\section{Robotic System Implementation}

The first experiments and architectural design were made with the prototypes described in section 2. The final implementation include an artificial hand commercially available equipped with sensor array and various components on the market. The functionality of the control unit is the same with minor parameter adjustment for this model of hand.

A 5 degrees of freedom 10.25" median reach and $13 \mathrm{oz}$ lifting capacity robotic arm was used, with a range of motion per axis of 180 degrees. The arm is driven by 4 servo motors located in the base, in the "shoulder", in the "elbow" and in the "wrist".

As end effector for the arm the anthropomorphic MechTE Robot Hand was used, constructed of anodized aircraft aluminum, with 14 points of motion, 5 degrees of freedom, four fingers and thumb open/close. No special force or speed requirements were put on the system, as this is merely an experimental system designed to verify the adopted concepts.

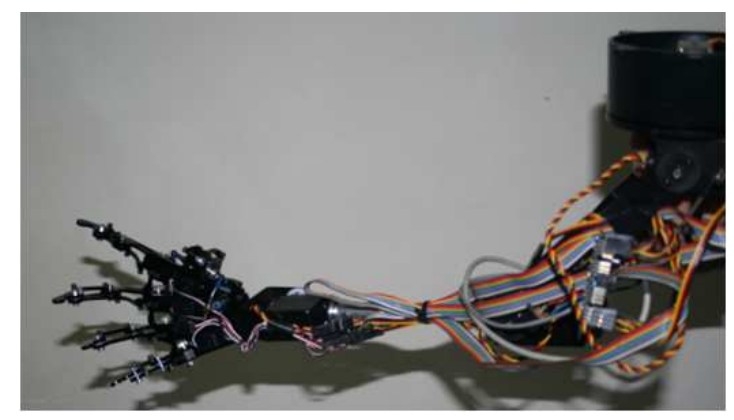

Fig. 5. Robotic arm and hand

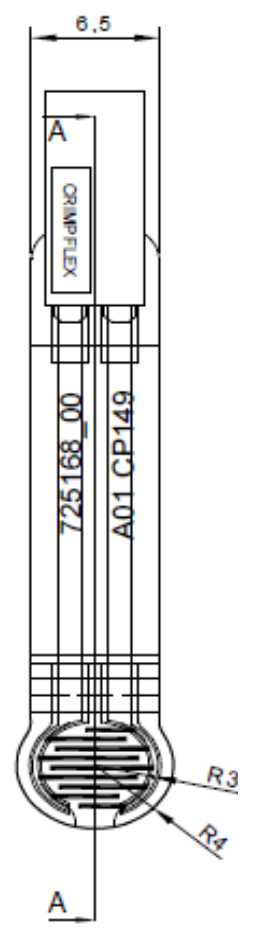

Fig. 6. Pressure sensor $\mathrm{CZN}-\mathrm{CP} 6$
To the robotic hand there was attached a pressure sensor array with 20 pressure sensors that send signals independently to the control unit, thus assuring the haptic feedback. The pressure sensors are of type CZN-CP6, depicted in fig. 6 , with a good response in the force domain similar to the force of a human hand.

The pressure sensors are distributed on the finger's phalanxes and on the palm pressure points, as can be seen in fig. 7 .

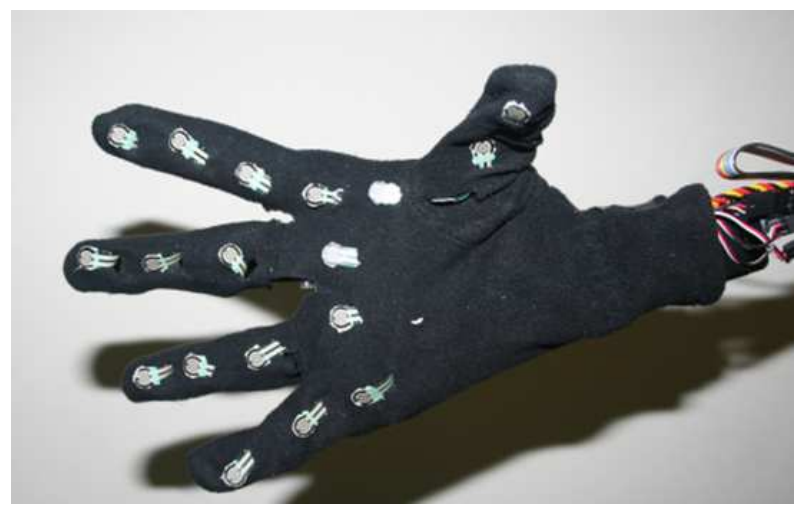

Fig. 7. Pressure sensor array

The Complex Interactive Control Glove shown in fig. 8 is worn by the operator and comprises the haptic feedback actuator array and the movement sensors for each finger. The type of glove used was 5DT Data Glove MRI.

The haptic actuators are of type Pico Vibe 308-100 8mm vibration motor and precision haptic $13 \mathrm{~mm}$ linear resonant actuators arranged in an array that duplicates the pressure sensor array on the robotic hand.

The signals from the movement sensors are preprocessed by a local control unit attached to the glove, which communicates with the central Control Unit.

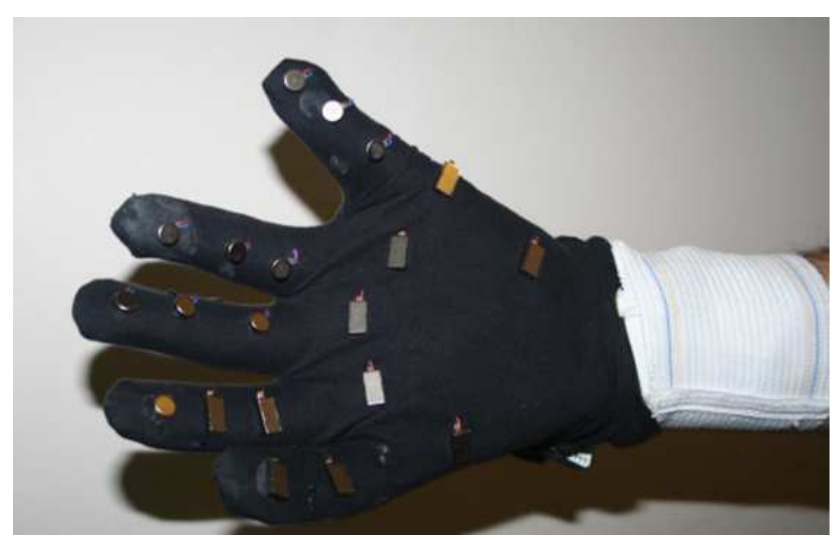

Fig. 8. Complex Interactive Control Glove

The movements of the operator arm is sensed by three joint sensor as presented in fig. 9. The joint sensors are build based on Flex Sensors that offer angle displacement measurement by bending with the robotic arm links. The resistance of the sensors varies proportional with the bending angle and have a very high life cycle. 


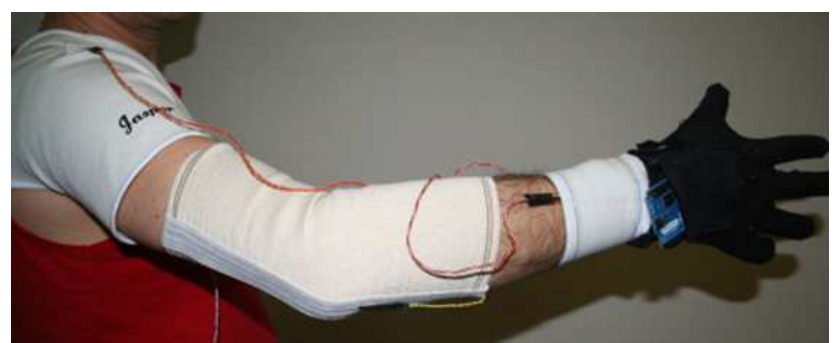

Fig. 9. Joint sensors

As mentioned above, the movements of the arm and hand can be controlled by a human operator, but also by the control unit independently or in collaboration.

\section{Experiments and Results}

The experiments conducted so far intended to validate the architecture and the processing algorithms. First issue addressed was if the robotic arm and hand really imitate the movements of the operator. Different combinations of movements, sequences of movements varying in distance and rotation were applied in order to verify the efficacy of the control method. The robotic arm and hand are moving as expected.

Relevant images are shown in fig. 10.
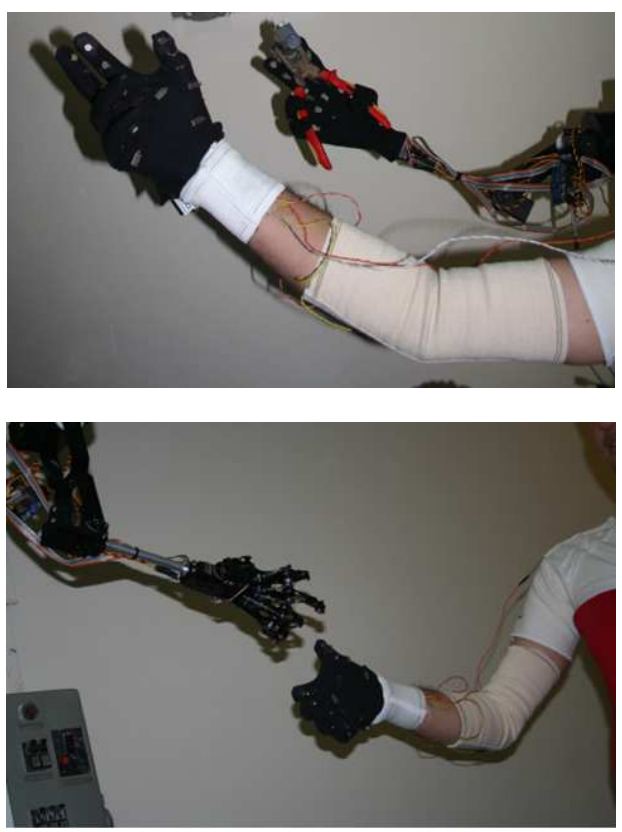

Fig. 10. Experiments conducted on the robotic system

The preliminary experiments conducted on the system showed promising results, as:

- The robotic hand and arm respond in real time to the operator's movements.

- The robotic hand and arm movements are controlled in range, force and speed according to the programmed safety limitations.

- The haptic feedback from the sensor array functions accurately providing the necessary data to the control unit and clear intuitive feedback to the operator.

- The control of the arm and hand are very intuitive and need no prior training.

- The accuracy of positioning is satisfactory and enables complex maneuvers, like handling pliers.

- The hand does not lose grip on the object during movements.

\section{Conclusions}

A robotic system comprising a robotic arm and an anthropomorphic hand that are remotely operated by a human operator and providing haptic feedback was designed and implemented. The system is suitable for hazardous environment applications, as the operator remains in a safe location performing complex human-specific handling operations by means of the robotic system.

The teleoperation function uses a Complex Interactive Control Glove and three joint sensors warn by the operator. The telepresence function is performed by an array of pressure sensors mounted on the robotic hand and an array of haptic actuators that convert the pressure in vibrations perceived by the operator's hand on the corresponding locations of the pressure sensors.

The experimental model proves the validity of the telepresence and teleoperation solutions that were adopted and also the control methods implemented in the control unit. The adopted control method is very intuitive and needs little prior training of the operator. The movement precision, the lag in the mechanical and the computer processing of the movement response have been experimentally found satisfactory and the robotic system is proven to be fully operational.

\section{Acknowledgement}

This work was supported by a grant of the Romanian National Authority for Scientific Research, Programme for research - Space Technology and Advanced Research - STAR, project number $82 / 2013$.

\section{References}

[1] S. Goto, Industrial Robotics: Theory, Modeling and Control (Force-free control for flexible motion of industrial articulated robot arm), Advanced Robotic Systems International, Chapter 30, pp. 813-840, Proliteratur Verlag, 2007.

[2] J. Richer and J. L. Drury, A Video Game - Based Framework for Analyzing Human - Robot Interaction, in proceeding s of the ACM conference on Human - Robot Interaction - HRI '06, 2006, pp . 266.

[3] D. Sakamoto, K. Honda, M. Inami, and T. Igarashi, Sketch and Run, in proceedings of the international conference on Human Factors in Computing Systems - CHI '09 , 2009, pp . 197.

[4] C. Glover, B. Russell, A. White, M. Miller, and A. Stoytchev, An Effective and Intuitive Control Interface for Remote Robot Teleoperation with Complete Haptic Feedback, in proceedings of the Emerging Technologies Conference - ETC, 2009. 
[5] S. Goto, Advances in Robot Manipulators (Industrial Robotics: Teleoperation System of Industrial Articulated Robot Arms by Using Forcefree Control), Advanced Robotic Systems International, INTECH, 2010.

[6] S. Goto, T. Naka, Y. Matsuda and N. Egashira, Teleoperation System of Robot Arms Combined with Remote Control and Visual Servo Control, Proceedings of the SICE Annual Conference 2010, August 18-21, Taipei, Taiwan, 2010.

[7] Velagic, J., Coralic, M. and Hebibovic, M. (2004). The Remote Control of Robot Manipulator for Precise Time-Limited Complex Path Tracking, Proceedings of the IEEE International Conference on Mechatronics and Robotics (MechRob2004), Volume 2, September 13-15, Aachen, Germany, pp. 841-846
[8] D. Lee, and M.W. Spong, Passive Bilateral Teleoperation with Constant Time Delay, IEEE Transactions on Robotics and Automation, vol. 22, no.2, pp. 269-281, April 2006.

[9] J. Scholtz, J. Young, J. L. Drury, and H. A. Yanco, Evaluation of Human - Robot Interaction Awareness in Search and Rescue, in IEEE international conference on Robotics and Automation ICRA '04. , 2004, vol. 3, pp. 2327 - 2332

[10] J. L. Drury, J. Scholtz, and H. A. Yanco, Awareness in Human Robot Interactions, in IEEE international conference on Systems, Man and Cybernetics - SMC '03. , 2003, vol. 1, pp. $912-918$. 